Research Article

\title{
Research on a Novel Kernel Based Grey Prediction Model and Its Applications
}

\author{
Xin Ma \\ School of Science, Southwest University of Science and Technology, Mianyang, China \\ Correspondence should be addressed to Xin Ma; cauchy7203@gmail.com
}

Received 2 July 2016; Revised 23 October 2016; Accepted 14 November 2016

Academic Editor: Michele Betti

Copyright (c) 2016 Xin Ma. This is an open access article distributed under the Creative Commons Attribution License, which permits unrestricted use, distribution, and reproduction in any medium, provided the original work is properly cited.

\begin{abstract}
The discrete grey prediction models have attracted considerable interest of research due to its effectiveness to improve the modelling accuracy of the traditional grey prediction models. The autoregressive $\operatorname{GM}(1,1)$ model, abbreviated as $\operatorname{ARGM}(1,1)$, is a novel discrete grey model which is easy to use and accurate in prediction of approximate nonhomogeneous exponential time series. However, the $\operatorname{ARGM}(1,1)$ is essentially a linear model; thus, its applicability is still limited. In this paper a novel kernel based $\operatorname{ARGM}(1,1)$ model is proposed, abbreviated as $\operatorname{KARGM}(1,1)$. The $\operatorname{KARGM}(1,1)$ has a nonlinear function which can be expressed by a kernel function using the kernel method, and its modelling procedures are presented in details. Two case studies of predicting the monthly gas well production are carried out with the real world production data. The results of $\operatorname{KARGM}(1,1)$ model are compared to the existing discrete univariate grey prediction models, including $\operatorname{ARGM}(1,1), \operatorname{NDGM}(1,1, k), \operatorname{DGM}(1,1)$, and NGBMOP, and it is shown that the $\operatorname{KARGM}(1,1)$ outperforms the other four models.
\end{abstract}

\section{Introduction}

The idea of the "Grey Box" modelling is trying to combine the advantages of the "White Box" and the "Black Box." Deng [1] has pioneered the Grey System Theory based on this idea. The grey prediction models play an important role in the Grey System Theory, and because of their effectiveness in time series prediction the grey prediction models have been widely adopted [2-5].

Over three decades of development, many new grey prediction models have been put forward, such as the $\operatorname{FGM}(1,1)$ [6], $\operatorname{DGMD}(1,1,1)$ [7], $\operatorname{NGM}(1,1, k)$ [8], and $\operatorname{SAGM}(1,1)[9]$. Along with these new models, some novel methodologies have also been proposed, and the discrete modelling technique is one of the most efficient methods to build the grey prediction models. The discrete modelling technique has been developed from the research of the $\operatorname{DGM}(1,1)$ model $[10]$, which is based on the basic $\operatorname{GM}(1,1)$ model. This novel technique has also been used to build the $\operatorname{NDGM}(1,1, k)$ model based on the $\operatorname{NGM}(1,1, k)[11]$. And in our previous works, this technique has been extended to build the discrete $\operatorname{GM}(1, n)$ models $[12,13]$. In these works, the discrete modelling technique has been proved efficient to improve the accuracy of the grey prediction models. Some novel grey prediction models for the nonlinear sequences are developed in recent years. For the univariate regression problems, the nonlinear grey Bernoulli model (NGBM) has been proposed by Chen et al. [14], which is more flexible than the existing grey prediction models and efficient to predict various time series. The NGBM model has attracted considerable research, and some improved grey prediction models based on it have been proposed, such as the Nash NGBM [15], the NGBM with optimal parameter [16], and the optimized NGBM [17] model. As for the multivariate regression problems, the nonlinear $\operatorname{GMC}(1, n)$ model $[18]$ has been proved to be more efficient to predict the nonlinear series than the existing models.

In recent researches, a novel grey prediction model directly built on the original series has been proposed, which is called the $\operatorname{DDGM}(1,1)$ model $[19]$ and is also called the autoregressive $\operatorname{GM}(1,1)$ model $(\operatorname{ARGM}(1,1))[20]$ as it is essentially in the autoregressive formulation. It is unnecessary to use the 1-AGO when building the $\operatorname{ARGM}(1,1)$ model; thus it is very easy to use. The $\operatorname{ARGM}(1,1)$ model has been proved to coincidence with the nonhomogeneous exponential law [19], and it has also been presented to be more efficient than 
the $\operatorname{DGM}(1,1)$ model in some applications $[19,20]$. However, the $\operatorname{ARGM}(1,1)$ model is essentially a linear model; thus its applicability is limited.

In order to improve the applicability of the $\operatorname{ARGM}(1,1)$ model, we use the kernel method to build a novel kernel based $\operatorname{ARGM}(1,1)$ model, abbreviated as $\operatorname{KARGM}(1,1)$. The kernel method has been developed from the Vapnik's Support Vector Machines (SVM) [21], and it has been proved to be very efficient to convert the classical linear models into nonlinear models in the previous researches [22-24]. The researches of Vapnik's SVM [21] are the initial works of the kernel method. But the formulation of the Vapnik's SVM is not easy to use as it involves in a quadric problem with inequivalent constraints. Suykens and Vandewalle [25] have proposed a simplified formulation of kernel method involving a quadric problem with equivalent constraints, which can be converted into a linear system. And the formulation by Suykens and Vandewalle has been proved to be available to extend to linear models into nonlinear models as efficient as the formulation of Vapnik's SVM [26, 27]. As for the time series regression, the recurrent LS-SVM [28] is a typical model for the nonlinear univariate time series, which can be more easily used to predict the chaotic time series than the recurrent neural networks [29]. In this work, the kernel method by Suykens and Vandewalle will be used to build the $\operatorname{KARGM}(1,1)$ model.

The rest of this paper is organized as follows. Section 2 presents a brief overview of the existing $\operatorname{ARGM}(1,1)$ model; Section 3 presents the modelling procedures of the $\operatorname{KARGM}(1,1)$; two case studies of predicting the gas well production are presented in Section 4, and conclusions are drawn in Section 5.

\section{Overview of the Autoregressive GM $(1,1)$ Model}

With a given time series $\{x(1), x(2), \ldots, x(n)\}$, the autoregressive $\operatorname{GM}(1,1)(\operatorname{ARGM}(1,1))$ model is represented as the following linear difference equation [20]:

$$
x(k)=\alpha x(k-1)+\beta, \quad k=2,3, \ldots, n .
$$

The parameters $\alpha$ and $\beta$ can be obtained using the least squares method as follows:

$$
[\alpha, \beta]^{T}=\left(B^{T} B\right)^{-1} B^{T} Y
$$

where

$$
Y=\left(\begin{array}{c}
x(2) \\
x(3) \\
\vdots \\
x(n)
\end{array}\right)
$$

$$
B=\left(\begin{array}{cc}
x(1) & 1 \\
x(2) & 1 \\
\vdots & \vdots \\
x(n-1) & 1
\end{array}\right) .
$$

The solution of the $\operatorname{ARGM}(1,1)$ model can be obtained using the recursive method, which is given as the following discrete function:

$$
x(k)=\alpha^{k} x(1)+\frac{1-\alpha^{k}}{1-\alpha} \beta .
$$

The discrete function (4) is used to compute the predicted values.

\section{The Proposed KARGM $(1,1)$ Model}

In this section, the modelling procedures of the novel kernel based autoregressive $\operatorname{GM}(1,1)$ model, abbreviated as $\operatorname{KARGM}(1,1)$, will be presented.

3.1. Representation of the $\operatorname{KARGM}(1,1)$ Model. With a given time series $\{x(1), x(2), \ldots, x(n)\}$, the $\operatorname{KARGM}(1,1)$ model is represented as the following difference equation:

$$
x(k)=\alpha x(k-1)+w^{T} \varphi(k)+\beta,
$$

where $\varphi$ is a nonlinear mapping, which is defined as

$$
\varphi: R \longrightarrow \mathscr{F},
$$

and $\mathscr{F}$ is a higher dimensional feature space; $w$ is a vector in $\mathscr{F}$.

The linear combination $w^{T} \varphi(k)$ is a nonlinear function of $k$. For example, if we consider a nonlinear function

$$
g(k)=0.3 \sin (k * \pi)+\sqrt{k},
$$

and define the nonlinear mapping as

$$
\varphi(k)=[\sin (k * \pi), \sqrt{k}]^{T},
$$

and set $w=[0.3,1]^{T}$, then we have

$$
g(k)=w^{T} \varphi(k) .
$$

3.2. Parameters Estimation of the $\operatorname{KARGM}(1,1)$ Model. Being different from the $\operatorname{ARGM}(1,1)$ model, we cannot simply use the least squares method to estimate the parameters of the $\operatorname{KARGM}(1,1)$ model, because it is not always computationally feasible to find the formulation of the nonlinear mapping $\varphi[30]$. Firstly, we consider the regularized problem as follows:

$$
\begin{aligned}
& \min \quad \mathscr{J}(\alpha, w, \mathbf{e})=\frac{1}{2} \alpha^{2}+\frac{1}{2}\|w\|^{2}+\frac{\gamma}{2} \sum_{k=2}^{n} e_{k}^{2} \\
& \text { s.t. } \quad x(k)=\alpha x(k-1)+w^{T} \varphi(k)+\beta+e_{k},
\end{aligned}
$$


where $\gamma$ is the regularized parameter to balance the flatness of the fitting curve and the error bound, and $\|\cdot\|$ is the 2-norm. The optimization problem (10) can be solved using the KKT conditions, which have been presented in Appendix A.

We firstly define the Lagrangian function as

$$
\begin{aligned}
\mathscr{L}(\alpha, w, \mathbf{e} ; \boldsymbol{\lambda}) \\
=\mathscr{J}(\alpha, w, \mathbf{e}) \\
\quad-\sum_{k=2}^{n} \lambda_{k}\left\{\alpha x(k-1)+w^{T} \varphi(k)+\beta+e_{k}-x(k)\right\},
\end{aligned}
$$

where $\lambda_{k}$ is the Lagrangian multiplier. Then the KKT conditions can be given as

$$
\begin{aligned}
\frac{\partial \mathscr{L}}{\partial \alpha} & =0 \Longrightarrow \\
\alpha & =\sum_{k=2}^{n} \lambda_{k} x(k-1) \\
\frac{\partial \mathscr{L}}{\partial w} & =0 \Longrightarrow \\
w & =\sum_{k=2}^{n} \lambda_{k} \varphi(k) \\
\frac{\partial \mathscr{L}}{\partial \beta} & =0 \Longrightarrow \\
\sum_{k=2}^{n} \lambda_{k} & =0 \\
\frac{\partial \mathscr{L}}{\partial e_{k}} & =0 \Longrightarrow \\
\frac{e_{k}}{\partial \lambda_{k}} & =\lambda_{k} \gamma^{-1} \\
\frac{\partial \mathscr{L}}{x(k)} & =\alpha x(k-1)+w^{T} \varphi(k)+\beta+e_{k} .
\end{aligned}
$$

By eliminating the $\alpha, w$ and $e_{k}$, the KKT conditions can be converted to the following linear system:

$$
\left(\begin{array}{cc}
0 & \overrightarrow{1}_{n-1}^{T} \\
\overrightarrow{1}_{n-1} & \Omega+Q+\gamma^{-1} I_{n-1}
\end{array}\right)\left(\begin{array}{l}
\beta \\
\vec{\lambda}
\end{array}\right)=\left(\begin{array}{l}
0 \\
Y
\end{array}\right),
$$

where

$$
\begin{aligned}
\overrightarrow{1}_{n-1} & =[1,1, \ldots, 1]_{n-1}^{T}, \\
\vec{\lambda} & =\left[\lambda_{2}, \lambda_{3}, \ldots, \lambda_{n}\right]^{T}, \\
Y & =[x(2), x(3), \ldots, x(n)]^{T}, \\
Q_{i j} & =x(i) x(j), \\
\Omega_{i j} & =\varphi(i+1) \cdot \varphi(j+1),
\end{aligned}
$$

and $I_{n-1}$ and $n-1$ dimensional identity matrix with all the diagonal elements are 1 and others are zero. The inner product $\Omega_{i j}$ can be expressed using a kernel function which satisfies the Mercer's condition, that is,

$$
\Omega_{i j}=\varphi(i+1) \cdot \varphi(j+1)=K(i+1, j+1) .
$$

The Gaussian kernel is often employed, which is defined as

$$
K(i, j)=\exp \left\{-\frac{(i-j)^{2}}{2 \sigma^{2}}\right\} \text {, }
$$

where $\sigma$ is the kernel parameter.

The linear system (13) can be solved within the inner product (15) expressed by the Gaussian kernel (16), and then the parameter $\beta$ and the Lagrangian multipliers $\lambda_{k}$ can be obtained. The parameter $\alpha$ can then be computed using the first equation in the KKT conditions (12).

3.3. The Solution of the $\operatorname{KARGM}(1,1)$ Model. The KAR$\operatorname{GM}(1,1)$ model $(5)$ can be easily solved using the recursive method as follows:

$$
\begin{aligned}
x(k)= & \alpha x(k-1)+w^{T} \varphi(k)+\beta \\
= & \alpha\left[\alpha x(k-2)+w^{T} \varphi(k-1)+\beta\right]+w^{T} \varphi(k) \\
& +\beta=\alpha^{2} x(k-2)+\sum_{\tau=k-1}^{k} \alpha^{k-\tau}\left[w^{T} \varphi(\tau)+\beta\right] \\
= & \cdots=\alpha^{k-1} x(1)+\sum_{\tau=2}^{k} a^{k-\tau}\left[w^{T} \varphi(\tau)+\beta\right] .
\end{aligned}
$$

Noticing the second equation in the KKT conditions (12), which can be rewritten as $w=\sum_{j=2}^{n} \lambda_{j} \varphi(j)$, we have

$$
w^{T} \varphi(\tau)=\sum_{j=2}^{n} \lambda_{j} \varphi(j) \cdot \varphi(\tau)
$$

Using the inner product (15), we can rewrite the nonlinear function (18) as follows:

$$
w^{T} \varphi(\tau)=\sum_{j=2}^{n} \lambda_{j} K(j, \tau) .
$$

For convenience, we note the following discrete function:

$$
\phi(\tau)=w^{T} \varphi(\tau)+\beta=\sum_{j=2}^{n} \lambda_{j} K(j, \tau)+\beta .
$$

Then the solution (17) can be rewritten as

$$
x(k)=\alpha^{k-1} x(1)+\sum_{\tau=2}^{k} a^{k-\tau} \phi(\tau) .
$$

The discrete function (21) can be used to compute the predicted series. 
TABLE 1: Monthly gas production data of the B51 in Sichuan, China.

\begin{tabular}{lccccccc}
\hline Month & Gas production & Month & Gas production & Month & Gas production & Month & Gas production \\
\hline 1 & 90.2837 & 6 & 63.9314 & 11 & 34.2398 & 16 & 53.2518 \\
2 & 72.1744 & 7 & 30.8003 & 12 & 44.0861 & 17 & 50.3282 \\
3 & 54.7787 & 8 & 60.5955 & 13 & 49.6398 & 18 & 37.8982 \\
4 & 54.1369 & 9 & 58.2767 & 14 & 51.7017 & 19 & 36.4632 \\
5 & 64.6249 & 10 & 7.5851 & 15 & 44.5744 & 20 & 48.0127 \\
\hline
\end{tabular}

It should be noticed that we do not need to know the expression of the nonlinear mapping $\varphi$, because all the computational procedures of the $\operatorname{KARGM}(1,1)$ model only involve in the inner product $\varphi(i) \cdot \varphi(j)$, and it can be expressed by a proper kernel function.

Actually, the KARGM $(1,1)$ represents a dynamical system which contains a "White" part and a "Black" part. The "White" part is the linear recursion which is known a priori, and the "Black" part is the linear combination $w^{T} \varphi(k)$, which is expressed by the kernel function and finally determined by the raw data. So we can say that the $\operatorname{KARGM}(1,1)$ model is a real "Grey" model.

3.4. Summary of Computational Steps. The computational steps of the $\operatorname{KARGM}(1,1)$ model are summarized as follows.

Step 1. Select an appropriate kernel function $K(\cdot, \cdot)$ and the regularized parameter $\gamma$ in (10).

Step 2. Compute the parameters $\beta$ and $\lambda_{2}, \lambda_{3}, \ldots, \lambda_{n}$ by solving the linear system (13), and then compute the parameter $\alpha$ using the first equation in the KKT conditions (12).

Step 3. Compute the values of the predicted series using the discrete function (21).

\section{Applications}

Two case studies of predicting the gas well production are carried out in this section to validate the effectiveness of the $\operatorname{KARGM}(1,1)$ model. The monthly production data are collected from two gas wells in Sichuan, China. In order to compare the performance of the $\operatorname{KARGM}(1,1)$ model and other existing discrete grey models, the $\operatorname{ARGM}(1,1)$, $\operatorname{NDGM}(1,1, k)[11], \operatorname{DGM}(1,1)[10]$, and the nonlinear grey Bernoulli model with optimal parameter $i$ (NGBMOP) [16] are also applied in the case studies. The mean absolute percentage error is used as an overall measurement of accuracy of the prediction models, which is defined as

$$
\text { MAPE }=\frac{1}{n} \sum_{k=1}^{n}\left|\frac{\widehat{x}(k)-x(k)}{x(k)}\right| \times 100(\%),
$$

where $x(k)$ is the real value and $\widehat{x}(k)$ is the predicted value, respectively.

4.1. Case Study 1. The raw data used in case study 1 are collected from the gas well B51 in Sichuan, China. Twenty points

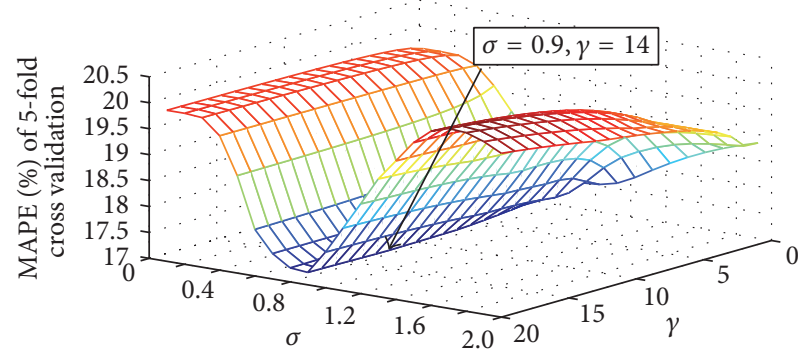

FIGURE 1: The MAPE of cross validation of $\operatorname{KARGM}(1,1)$ with different $\sigma$ and $\gamma$ using the 5-fold cross validation in Case Study 1.

of monthly gas production $\left(10^{4} \mathrm{~m}^{3}\right)$ are listed in Table 1 . The first 15 points are used to build the models, and the last 5 points are used for testing. The Gaussian kernel (16) is used to build the $\operatorname{KARGM}(1,1)$ model.

The kernel parameter $\sigma$ and the regularized parameter $\gamma$ are tuned using the cross validation, which is widely used in the other kernel methods, such as the Partially Linear LSSVM [31]. The 5-fold cross validation is employed in this case. The MAPE of validation is used to assess the chosen kernel parameter and the regularized parameter. A brief summary of the 5 -fold cross validation has been described in Appendix B. The results of the MAPE for each pair of $\sigma$ and $\gamma$ are plotted in Figure 1. It can be seen that the minimum MAPE is found at the point $\sigma=0.9$ and $\gamma=14$; thus this pair will be used to build the $\operatorname{KARGM}(1,1)$ in this case.

The predicted values by the $\operatorname{KARGM}(1,1), \operatorname{ARGM}(1,1)$, $\operatorname{NDGM}(1,1, k), \operatorname{DGM}(1,1)$, and $\operatorname{NGBMOP}$ are listed in Table 2, along with the absolute percentage error of each point and the MAPE of each model. The results are also plotted in Figure 2.

It can be seen in Table 2 that the minimum MAPE for fitting of $\operatorname{ARGM}(1,1), \operatorname{NDGM}(1,1, k), \operatorname{DGM}(1,1)$, and NBGMOP is more than three times larger than that of $\operatorname{KARGM}(1,1)$. The MAPE for prediction of $\operatorname{KARGM}(1,1)$ is much smaller than the $\operatorname{NDGM}(1,1, k), \operatorname{DGM}(1,1)$, and NBGMOP, and it is slightly larger than that of the $\operatorname{ARGM}(1,1)$. It is shown in Figure 2 that the predicted values of $\operatorname{KARGM}(1,1)$ are quite close to the real values, and these values are presented to follow the overall trend of the monthly production of B51; but the distances between the predicted values of the other four models are very large, and they have failed in catching the overall trend of the gas production, which indicates that the modelling accuracy of these four models is not acceptable. In summary, the $\operatorname{KARGM}(1,1)$ performs best in the presented models. 
TABLE 2: Predicted values of monthly gas production of $\operatorname{B51}$ by the $\operatorname{ARGM}(1,1), \operatorname{NDGM}(1,1, k), \operatorname{DGM}(1,1), \operatorname{NGBMOP}$, and $\operatorname{KARGM}(1,1)$.

\begin{tabular}{|c|c|c|c|c|c|c|c|c|c|c|c|}
\hline Month & Gas production & $\operatorname{ARGM}(1,1)$ & Error & $\operatorname{NDGM}(1,1, k)$ & Error & $\operatorname{DGM}(1,1)$ & Error & NGBMOP & Error & $\operatorname{KAGM}(1,1)$ & Error \\
\hline 1 & 90.2837 & 90.2837 & 0 & 90.2837 & 0 & 90.2837 & 0 & 90.2837 & 0.00 & 90.2837 & 0.00 \\
\hline 2 & 72.1744 & 59.1583 & 18.03 & 73.2911 & 1.55 & 63.5896 & 11.89 & 72.8510 & 0.94 & 70.0022 & 3.01 \\
\hline 3 & 54.7787 & 51.0645 & 6.78 & 64.7782 & 18.25 & 61.0010 & 11.36 & 65.2404 & 19.10 & 55.9178 & 2.08 \\
\hline 4 & 54.1369 & 48.9598 & 9.56 & 57.9739 & 7.09 & 58.5177 & 8.09 & 61.4602 & 13.53 & 53.5025 & 1.17 \\
\hline 5 & 64.6249 & 48.4125 & 25.09 & 52.6425 & 18.54 & 56.1356 & 13.14 & 58.5062 & 9.47 & 65.0060 & 0.59 \\
\hline 6 & 63.9314 & 48.2702 & 24.50 & 48.5808 & 24.01 & 53.8504 & 15.77 & 55.9700 & 12.45 & 61.2468 & 4.20 \\
\hline 7 & 30.8003 & 48.2332 & 56.60 & 45.6134 & 48.09 & 51.6582 & 67.72 & 53.6995 & 74.35 & 34.3068 & 11.38 \\
\hline 8 & 60.5955 & 48.2236 & 20.42 & 43.5893 & 28.07 & 49.5553 & 18.22 & 51.6200 & 14.81 & 59.2332 & 2.25 \\
\hline 9 & 58.2767 & 48.2211 & 17.25 & 42.3785 & 27.28 & 47.5380 & 18.43 & 49.6887 & 14.74 & 55.7955 & 4.26 \\
\hline 10 & 7.5851 & 48.2204 & 535.73 & 41.8686 & 451.98 & 45.6028 & 501.22 & 47.8783 & 531.21 & 12.5928 & 66.02 \\
\hline 11 & 34.2398 & 48.2202 & 40.83 & 41.9629 & 22.56 & 43.7464 & 27.76 & 46.1701 & 34.84 & 32.4912 & 5.11 \\
\hline 12 & 44.0861 & 48.2202 & 9.38 & 42.5780 & 3.42 & 41.9656 & 4.81 & 44.5509 & 1.05 & 45.1528 & 2.42 \\
\hline 13 & 49.6398 & 48.2202 & 2.86 & 43.6422 & 12.08 & 40.2572 & 18.90 & 43.0106 & 13.35 & 49.4121 & 0.46 \\
\hline 14 & 51.7017 & 48.2202 & 6.73 & 45.0933 & 12.78 & 38.6184 & 25.31 & 41.5412 & 19.65 & 51.3527 & 0.68 \\
\hline 15 & 44.5744 & 48.2202 & 8.18 & 46.8780 & 5.17 & 37.0463 & 16.89 & 40.1364 & 9.96 & 45.2044 & 1.41 \\
\hline MAPE & & & 52.13 & & 45.39 & & 50.63 & & 51.30 & & 7.00 \\
\hline 16 & 53.2518 & 48.2202 & 9.45 & 48.9502 & 8.08 & 35.5382 & 33.26 & 38.7911 & 27.16 & 47.2950 & 11.19 \\
\hline 17 & 50.3282 & 48.2202 & 4.19 & 51.2703 & 1.87 & 34.0915 & 32.26 & 37.5008 & 25.49 & 50.3266 & 0.00 \\
\hline 18 & 37.8982 & 48.2202 & 27.24 & 53.8041 & 41.97 & 32.7037 & 13.71 & 36.2619 & 4.32 & 50.5842 & 33.47 \\
\hline 19 & 36.4632 & 48.2202 & 32.24 & 56.5221 & 55.01 & 31.3724 & 13.96 & 35.0710 & 3.82 & 50.5825 & 38.72 \\
\hline 20 & 48.0127 & 48.2202 & 0.43 & 59.3988 & 23.71 & 30.0953 & 37.32 & 33.9254 & 29.34 & 50.5832 & 5.35 \\
\hline MAPE & & & 14.71 & & 26.13 & & 26.10 & & 18.02 & & 17.75 \\
\hline
\end{tabular}

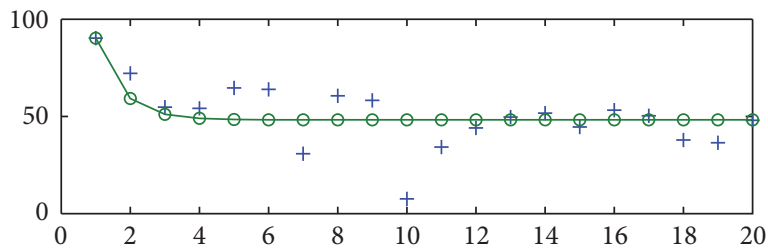

(a)

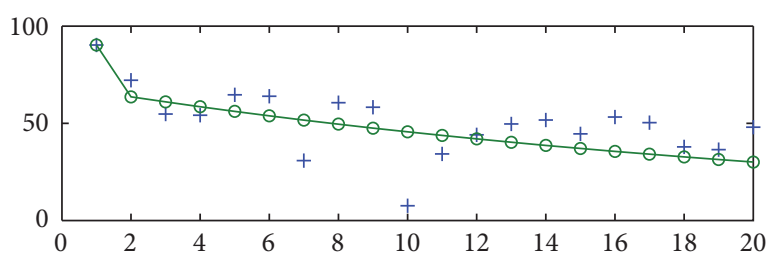

(c)

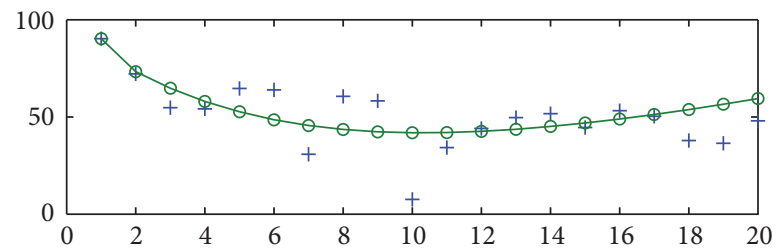

(b)

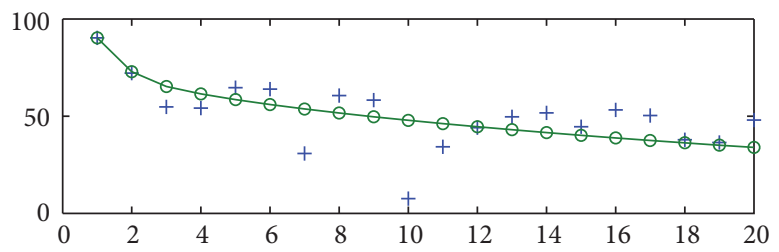

(d)

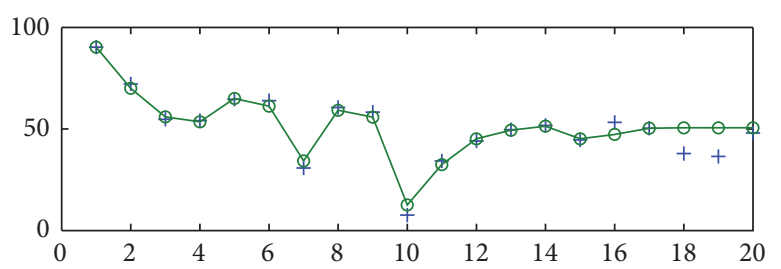

(e)

FIGURE 2: Prediction results of the monthly gas production of B51 by the prediction models. The raw data are plotted using the mark "+" and the predicted values are plotted using the solid line and the mark "o". (a) $\operatorname{ARGM}(1,1)$; (b) $\operatorname{NDGM}(1,1, k) ;(\mathrm{c}) \operatorname{DGM}(1,1) ;(\mathrm{d}) \mathrm{NGBMOP}$; (e) $\operatorname{KARGM}(1,1)$. 
TABLE 3: Monthly gas production data of the B41 in Sichuan, China.

\begin{tabular}{|c|c|c|c|c|c|c|c|}
\hline Month & Gas production & Month & Gas production & Month & Gas production & Month & Gas production \\
\hline 1 & 12.8 & 6 & 9.3 & 11 & 12.2 & 16 & 7.5 \\
\hline 2 & 9.2 & 7 & 10.3 & 12 & 11 & 17 & 6.8 \\
\hline 3 & 8.8 & 8 & 9.4 & 13 & 9 & 18 & 7.5 \\
\hline 4 & 9.2 & 9 & 9.3 & 14 & 8.2 & 19 & 7.7 \\
\hline 5 & 8.5 & 10 & 11.2 & 15 & 6.4 & 20 & 8.5 \\
\hline
\end{tabular}

TABLE 4: Predicted values of monthly gas production of $\mathrm{B} 41$ by the $\operatorname{ARGM}(1,1), \operatorname{NDGM}(1,1, k), \operatorname{DGM}(1,1), \operatorname{NGBMOP}$, and $\operatorname{KARGM}(1,1)$.

\begin{tabular}{|c|c|c|c|c|c|c|c|c|c|c|c|}
\hline Month & Gas production & $\operatorname{ARGM}(1,1)$ & Error & $\operatorname{NDGM}(1,1, k)$ & Error & $\operatorname{DGM}(1,1)$ & Error & NGBMOP & Error & $\operatorname{KAGM}(1,1)$ & Error \\
\hline 1 & 12.8 & 12.8 & 0 & 12.8 & 0 & 12.8 & 0 & 12.8 & 0 & 12.8 & 0.00 \\
\hline 2 & 9.2 & 10.83 & 17.75 & 7.95 & 13.60 & 9.60 & 4.35 & 9.09 & 1.19 & 9.22 & 0.21 \\
\hline 3 & 8.8 & 9.89 & 12.33 & 8.56 & 2.70 & 9.57 & 8.79 & 9.41 & 6.91 & 8.88 & 0.96 \\
\hline 4 & 9.2 & 9.43 & 2.48 & 9.11 & 0.99 & 9.55 & 3.77 & 9.50 & 3.23 & 8.80 & 4.33 \\
\hline 5 & 8.5 & 9.21 & 8.33 & 9.58 & 12.72 & 9.52 & 12.00 & 9.53 & 12.15 & 9.00 & 5.89 \\
\hline 6 & 9.3 & 9.10 & 2.13 & 9.97 & 7.20 & 9.49 & 2.08 & 9.54 & 2.61 & 9.41 & 1.22 \\
\hline 7 & 10.3 & 9.05 & 12.13 & 10.26 & 0.38 & 9.47 & 8.08 & 9.54 & 7.40 & 9.60 & 6.81 \\
\hline 8 & 9.4 & 9.03 & 3.98 & 10.44 & 11.11 & 9.44 & 0.44 & 9.52 & 1.31 & 9.54 & 1.52 \\
\hline 9 & 9.3 & 9.01 & 3.07 & 10.50 & 12.96 & 9.41 & 1.23 & 9.50 & 2.17 & 9.93 & 6.76 \\
\hline 10 & 11.2 & 9.01 & 19.57 & 10.43 & 6.91 & 9.39 & 16.17 & 9.47 & 15.41 & 10.95 & 2.21 \\
\hline 11 & 12.2 & 9.01 & 26.18 & 10.19 & 16.48 & 9.36 & 23.26 & 9.44 & 22.59 & 11.64 & 4.60 \\
\hline 12 & 11 & 9.00 & 18.14 & 9.77 & 11.14 & 9.34 & 15.12 & 9.41 & 14.46 & 11.04 & 0.35 \\
\hline 13 & 9 & 9.00 & 0.04 & 9.16 & 1.74 & 9.31 & 3.45 & 9.37 & 4.14 & 9.41 & 4.59 \\
\hline 14 & 8.2 & 9.00 & 9.80 & 8.31 & 1.34 & 9.28 & 13.22 & 9.33 & 13.83 & 7.76 & 5.42 \\
\hline 15 & 6.4 & 9.00 & 40.68 & 7.20 & 12.55 & 9.26 & 44.67 & 9.29 & 45.21 & 6.78 & 5.94 \\
\hline MAPE & & & 11.77 & & 7.45 & & 10.44 & & 10.17 & & 3.39 \\
\hline 16 & 7.5 & 9.00 & 20.05 & 5.80 & 22.63 & 9.23 & 23.10 & 9.25 & 23.36 & 6.65 & 11.34 \\
\hline 17 & 6.8 & 9.00 & 32.40 & 4.07 & 40.17 & 9.21 & 35.40 & 9.21 & 35.42 & 7.14 & 5.07 \\
\hline 18 & 7.5 & 9.00 & 20.05 & 1.96 & 73.90 & 9.18 & 22.42 & 9.16 & 22.20 & 7.86 & 4.78 \\
\hline 19 & 7.7 & 9.00 & 16.93 & -0.58 & 107.55 & 9.16 & 18.91 & 9.12 & 18.44 & 8.44 & 9.61 \\
\hline 20 & 8.5 & 9.00 & 5.92 & -3.60 & 142.40 & 9.13 & 7.42 & 9.07 & 6.76 & 8.77 & 3.14 \\
\hline MAPE & & & 19.07 & & 77.33 & & 21.45 & & 21.24 & & 6.79 \\
\hline
\end{tabular}

4.2. Case Study 2. The raw data used in case study 2 are collected from the gas well B41 in Sichuan, China. Twenty points of monthly gas production $\left(10^{4} \mathrm{~m}^{3}\right)$ are listed in Table 3. The first 15 points are used to build the models, and the last 5 points are used for testing. The Gaussian kernel (16) is also used to build the $\operatorname{KARGM}(1,1)$ model.

In this case, the kernel parameter $\sigma$ and the regularized parameter $\gamma$ are tuned using 5-fold cross validation, and the results of the MAPE for each pair of $\sigma$ and $\gamma$ are plotted in Figure 4. The minimum MAPE is found at the point $\sigma=$ 2.0 and $\gamma=11$; thus this pair will be used to build the $\operatorname{KARGM}(1,1)$ in this case.

The predicted values by the $\operatorname{KARGM}(1,1), \operatorname{ARGM}(1,1)$, $\operatorname{NDGM}(1,1, k), \operatorname{DGM}(1,1)$, and $\operatorname{NBGMOP}$ are listed in Table 4, along with the absolute percentage error of each point and the MAPE of each model. The results are also plotted in Figure 3.

It can be seen in Table 4 that the minimum MAPE for fitting of $\operatorname{ARGM}(1,1), \operatorname{NDGM}(1,1, k), \operatorname{DGM}(1,1)$, and NBGMOP is more than two times larger than that of $\operatorname{KARGM}(1,1)$. The minimum MAPE for prediction of
$\operatorname{ARGM}(1,1), \operatorname{NDGM}(1,1, k), \operatorname{DGM}(1,1)$, and NBGMOP is more than three times larger than that of the $\operatorname{KARGM}(1,1)$. In Figure 3 it is also shown that the predicted values of $\operatorname{KARGM}(1,1)$ are very close to the real values and the $\operatorname{KARGM}(1,1)$ can accurately catch the overall trend of the monthly production of B41; but the distances between the predicted values of the other four models are still very large. In this case study it is clearly that the $\operatorname{KARGM}(1,1)$ has be best performance.

\section{Conclusions}

In this paper, a novel kernel based grey model (KAR$\operatorname{GM}(1,1))$ has been proposed, and its effectiveness has been assessed in the two case studies of the gas well production forecasting in comparison to the existing discrete grey models, including the $\operatorname{ARGM}(1,1), \operatorname{NDGM}(1,1, k), \operatorname{DGM}(1,1)$, and NBGMOP. The results of the case studies have shown that the novel $\operatorname{KARGM}(1,1)$ model outperformed the other three discrete grey models. 


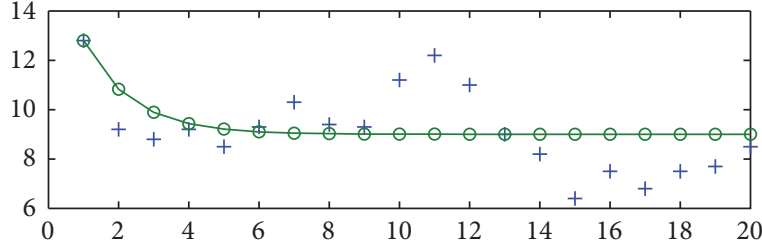

(a)

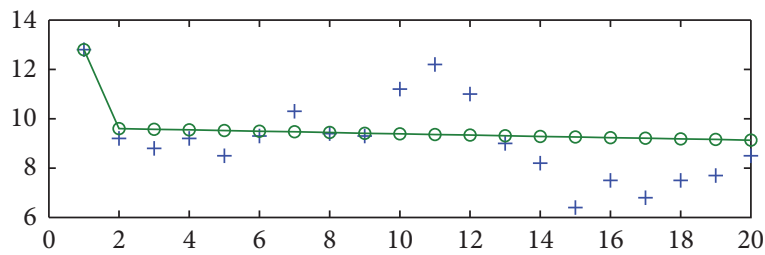

(c)

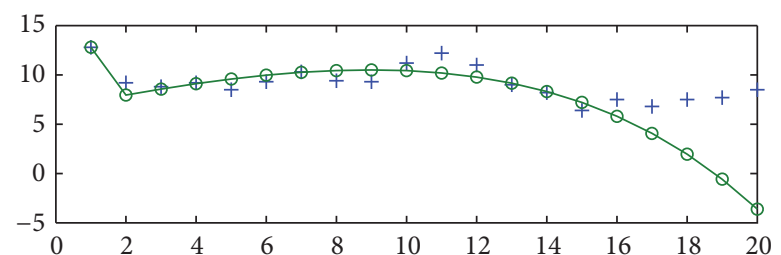

(b)

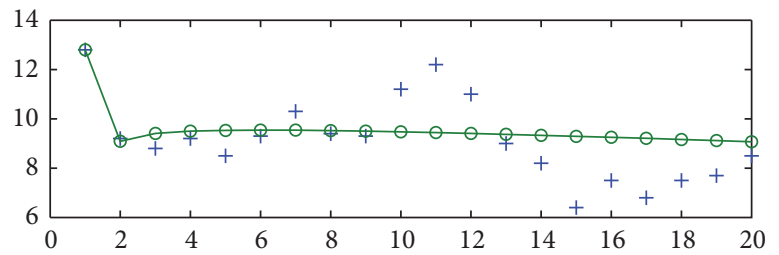

(d)

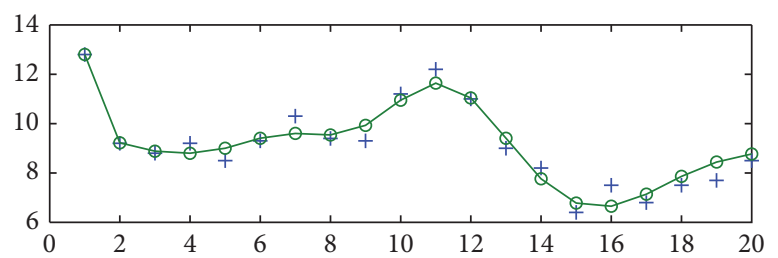

(e)

FIGURE 3: Prediction results of the monthly gas production of B41 by the prediction models. The raw data are plotted using the mark " + " and the predicted values are plotted using the solid line and the mark "o". (a) $\operatorname{ARGM}(1,1) ;($ b) NDGM $(1,1, k) ;(c) \operatorname{DGM}(1,1) ;(d) \operatorname{NGBMOP} ;(\mathrm{e})$ $\operatorname{KARGM}(1,1)$.

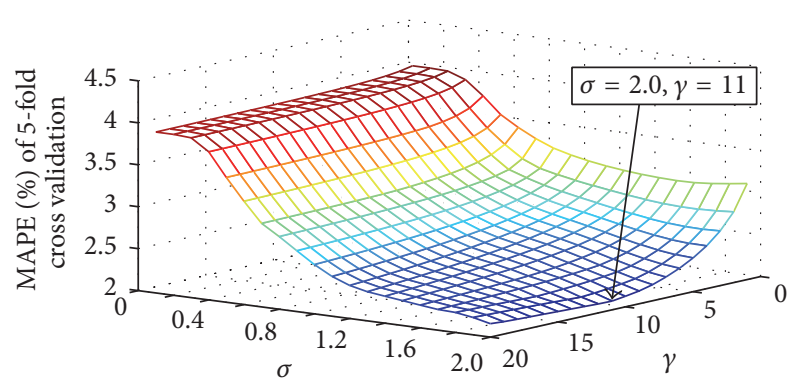

FIgURE 4: The MAPE of cross validation of $\operatorname{KARGM}(1,1)$ with different $\sigma$ and $\gamma$ using the 5-fold cross validation in Case Study 2.

Essentially, the existing $\operatorname{ARGM}(1,1), \operatorname{NDGM}(1,1, k)$, and $\operatorname{DGM}(1,1)$ are linear model, and their solutions are all exponential functions. And this is also the reason why they only produce simple curves in the case studies, which cannot be used to express complex time series. The NBGMOP is a nonlinear model, but its applicability is still limited according to the results shown in the case studies. On the other hand, the $\operatorname{KARGM}(1,1)$ model contains a general nonlinear function $w^{T} \varphi$, which is expressed by a proper kernel function as shown in (19) and determined by the raw data. Thus the $\operatorname{KARGM}(1,1)$ model can be more effective to deal with complex time series.

What is more, this paper has illustrated a new way of building the discrete grey model using the kernel method and also proved the effectiveness of this new methodology. Thus, the relative researches can be carried out based on this research in the future.

\section{Appendix}

\section{A. The KKT Conditions for Equality Constrained Convex Minimization}

Consider the equality constrained optimization problem

$$
\begin{array}{ll}
\min & f(x) \\
\text { s.t. } & h_{j}(x)=0, \quad j=1,2, \ldots, m,
\end{array}
$$

where the objective function $f$ and the constraint functions $h_{j}(x)=0(j=1,2, \ldots, m)$ are supposed to be continuously differentiable at point $x^{*}$. One defines the Lagrangian function as

$$
\mathscr{L}(x ; \lambda)=f(x)-\sum_{j=1}^{m} \lambda_{j} h_{j}(x),
$$

where the $\lambda=\left[\lambda_{1}, \ldots, \lambda_{m}\right]^{T}$ are the Lagrangian multipliers. The point $x^{*}$ and the Lagrangian multipliers must satisfy the KKT conditions if the point $x^{*}$ is a local minimum. In this case, the KarushCKuhnCTucker (KKT) conditions can be interpreted using the Lagrangian function as

$$
\begin{aligned}
& \frac{\partial \mathscr{L}(x ; \lambda)}{\partial x}=0 \\
& \frac{\partial \mathscr{L}(x ; \lambda)}{\partial \lambda_{j}}=0, \quad j=1,2, \ldots, m .
\end{aligned}
$$


The KKT conditions are sufficient and necessary to the optimization problem (A.1) if the objective function $f(x)$ is convex and the constrained functions $h_{j}(x)=0(j=$ $1,2, \ldots, m)$ are linear functions. In this case the local minimum $x^{*}$ which satisfies the KKT conditions is a global minimum; that is, the solution of the minimization problem (A.1) is equivalent to the solution of the KKT conditions (A.3). (More details of the KKT conditions can be seen in [32], pages: 243-245.)

\section{B. A Brief Summary of the 5-Fold Cross Validation Used in the Case Studies}

With a given time series $\{x(1), x(2), \ldots, x(n)\}$, the 5 -fold cross validation can be described as follows.

Step 1. Divide the original time series into 5 subsets randomly, and mark them as $S_{1}, S_{2}, \ldots, S_{5}$.

Step 2. Build the $\operatorname{KARGM}(1,1)$ model using four subsets and the rest subset is used for validation. (For example, the first time, the $\operatorname{KARGM}(1,1)$ model is built on the subset $S_{1}, S_{2}, S_{3}, S_{4}$, and the subset $S_{5}$ is used for validation. This procedure will be repeated for 5 times, and all the subset will be used for validation.) Compute the MAPE for all the validation procedures.

Step 3. For $\sigma=0.1,0.2, \ldots, 2$ and $\sigma=1,2, \ldots, 20$, repeat Step 2 and store the values of the MAPE with all the pairs of $\sigma$ and $\gamma$.

Step 4. Output the values of optimal $\sigma$ and $\gamma$ corresponding to the minimum MAPE.

\section{Competing Interests}

The author declares that there is no conflict of interests regarding the publication of this paper.

\section{Acknowledgments}

This work is supported by the Doctoral Research Foundation of Southwest University of Science and Technology (no. 16zx7140).

\section{References}

[1] J.-L. Deng, "Control problems of grey systems," Systems \& Control Letters, vol. 1, no. 5, pp. 288-294, 1982.

[2] X. Ma, D. Luo, X.-F. Ding, and J.-J. Zhou, "An algorithm based on the GM(1,1) model on increasing oil production of measures operation for a single well," in Proceedings of the 24th IEEE International Conference on Grey Systems and Intelligent Services (GSIS '13), pp. 158-160, Macao, China, November 2013.

[3] X. Ma and Z.-B. Liu, "Predicting the cumulative oil field production using the novel grey ENGM model," Journal of Computational and Theoretical Nanoscience, vol. 13, no. 1, pp. 89-95, 2016.
[4] D. Chenyan, Z. Songtao, and D. Julong, "Numerical mapping in DNA sequences and analysis of the genetic information by GM (1, N)," Journal of Grey System, vol. 24, no. 3, 2012.

[5] Q.-F. Li, Y.-G. Dang, and Z.-X. Wang, "Analysis of the regional coordination development systems based on GRA and GM(1,N)," Journal of Grey System, vol. 24, no. 1, pp. 95-100, 2012.

[6] T. L. Tien, "A new grey prediction model FGM $(1,1)$," Mathematical and Computer Modeling, vol. 49, no. 7-8, pp. 1416-1426, 2009.

[7] C.-K. Chen and T.-L. Tien, "The indirect measurement of tensile strength by the deterministic grey dynamic model DGDM $(1,1$, 1)," International Journal of Systems Science, vol. 28, no. 7, pp. 683-690, 1997.

[8] J. Cui, S.-F. Liu, B. Zeng, and N.-M. Xie, "A novel grey forecasting model and its optimization," Applied Mathematical Modelling, vol. 37, no. 6, pp. 4399-4406, 2013.

[9] D. Q. Truong and K. K. Ahn, "An accurate signal estimator using a novel smart adaptive grey model SAGM(1,1)," Expert Systems with Applications, vol. 39, no. 9, pp. 7611-7620, 2012.

[10] N.-M. Xie and S.-F. Liu, "Discrete grey forecasting model and its optimization," Applied Mathematical Modelling, vol. 33, no. 2, pp. 1173-1186, 2009.

[11] N.-M. Xie, S.-F. Liu, Y.-J. Yang, and C.-Q. Yuan, "On novel grey forecasting model based on non-homogeneous index sequence," Applied Mathematical Modelling, vol. 37, no. 7, pp. 5059-5068, 2013.

[12] X. Ma and Z. Liu, "Predicting the oil field production using the novel discrete GM(1,N) model," Journal of Grey System, vol. 27, no. 4, pp. 63-73, 2015.

[13] X. Ma and Z.-B. Liu, "Research on the novel recursive discrete multivariate grey prediction model and its applications," Applied Mathematical Modelling, vol. 40, no. 7-8, pp. 4876-4890, 2016.

[14] C.-I. Chen, H. L. Chen, and S.-P. Chen, "Forecasting of foreign exchange rates of Taiwan's major trading partners by novel nonlinear Grey Bernoulli model $\operatorname{NGBM}(1,1)$," Communications in Nonlinear Science and Numerical Simulation, vol. 13, no. 6, pp. 1194-1204, 2008.

[15] H.-T. Pao, H.-C. Fu, and C.-L. Tseng, "Forecasting Taiwans major stock indices by the Nash nonlinear grey Bernoulli model," Energy, vol. 40, no. 1, pp. 400-409, 2012.

[16] H.-T. Pao, H.-C. Fu, and C.-L. Tseng, "Forecasting of $\mathrm{CO}_{2}$ emissions, energy consumption and economic growth in China using an improved grey model," Energy, vol. 40, no. 1, pp. 400409, 2012.

[17] Z.-X. Wang, K. W. Hipel, Q. Wang, and S.-W. He, "An optimized $\operatorname{NGBM}(1,1)$ model for forecasting the qualified discharge rate of industrial wastewater in China," Applied Mathematical Modelling, vol. 35, no. 12, pp. 5524-5532, 2011.

[18] Z.-X. Wang, "Nonlinear grey prediction model with convolution integral NGMC $(1, n)$ and its application to the forecasting of China's industrial $\mathrm{SO}_{2}$ emissions," Journal of Applied Mathematics, vol. 2014, Article ID 580161, 9 pages, 2014.

[19] B. Zeng and S.-F. Liu, "Direct modeling approach of DGM (1, 1) with approximate non-homogeneous exponential sequence," System Engineering Theory and Practice, vol. 31, no. 2, pp. 297301, 2011.

[20] L. Wu, S. Liu, H. Chen, and N. Zhang, "Using a novel grey system model to forecast natural gas consumption in China," Mathematical Problems in Engineering, vol. 2015, Article ID 686501, 7 pages, 2015. 
[21] V. N. Vapnik, Statistical Learning Theory, Wiley-Interscience, 1998.

[22] B. Schölkopf, A. Smola, and K. Müller, "Kernel principal component analysis," in Proceedings of the 7th International Conference on Artificial Neural Networks (ICANN '97), pp. 583588, Springer, Lausanne, Switzerland, 1997.

[23] B. Scholkopft and K.-R. Mullert, "Fisher discriminant analysis with kernels," in Proceedings of the Neural Networks for Signal Processing IX, vol. 1, pp. 41-48, August 1999.

[24] M. E. Tipping, "Sparse Bayesian learning and the relevance vector machine," The Journal of Machine Learning Research, vol. 1, no. 3, pp. 211-244, 2001.

[25] J. A. K. Suykens and J. Vandewalle, "Least squares support vector machine classifiers," Neural Processing Letters, vol. 9, no. 3, pp. 293-300, 1999.

[26] T. Van Gestel, J. A. K. Suykens, G. Lanckriet, A. Lambrechts, B. De Moor, and J. Vandewalle, "Bayesian framework for leastsquares support vector machine classifiers, gaussian processes, and kernel fisher discriminant analysis," Neural Computation, vol. 14, no. 5, pp. 1115-1147, 2002.

[27] J. A. K. Suykens, T. Van Gestel, J. Vandewalle, and B. De Moor, "A support vector machine formulation to PCA analysis and its kernel version," IEEE Transactions on Neural Networks, vol. 14, no. 2, pp. 447-450, 2003.

[28] J. A. K. Suykens and J. Vandewalle, "Recurrent least squares support vector machines," IEEE Transactions on Circuits and Systems I: Fundamental Theory and Applications, vol. 47, no. 7, pp. 1109-1114, 2000.

[29] L. R. Medsker and L. C. Jain, Recurrent Neural Networks: Design and Applications, CRC Press, Boca Raton, Fla, USA, 2001.

[30] A. J. Smola and B. Schölkopf, "A tutorial on support vector regression," Statistics and Computing, vol. 14, no. 3, pp. 199-222, 2004.

[31] M. Espinoza, J. A. K. Suykens, and B. De Moor, "Partially linear models and least squares support vector machines," in Proceedings of the 43rd IEEE Conference on Decision and Control (CDC '04), vol. 4, pp. 3388-3393, IEEE, December 2004.

[32] S. Boyd and L. Vandenberghe, Convex Optimization, Cambridge University Press, 2004. 


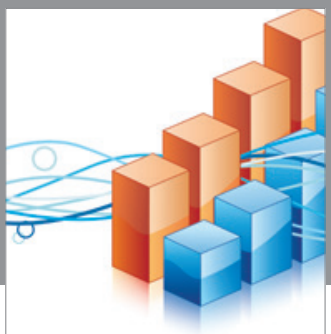

Advances in

Operations Research

vatem alat4

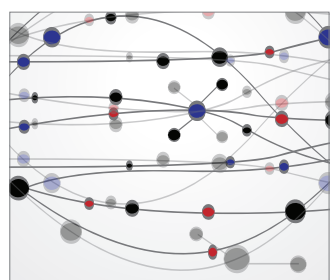

\section{The Scientific} World Journal
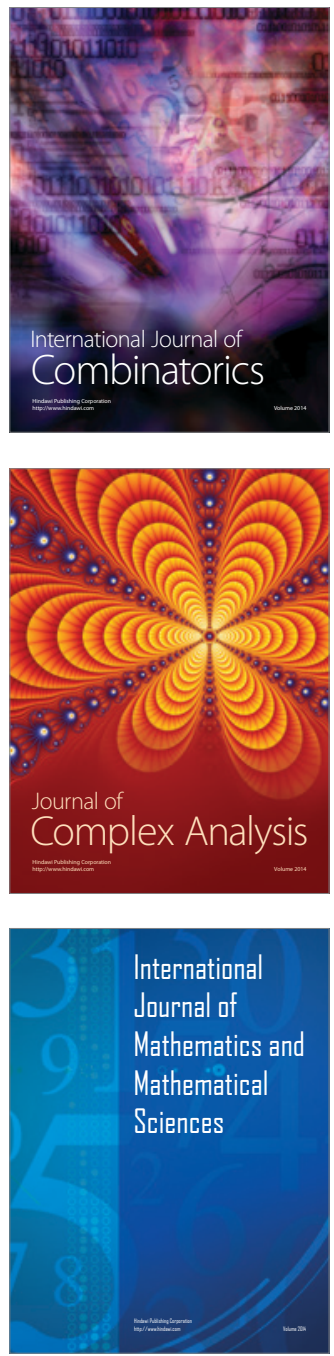
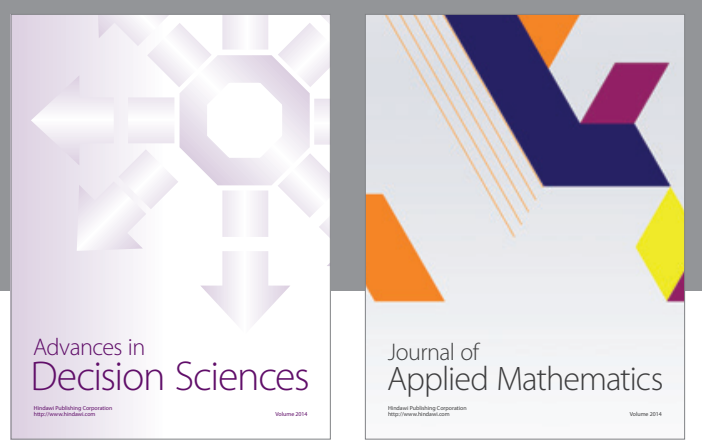

Algebra

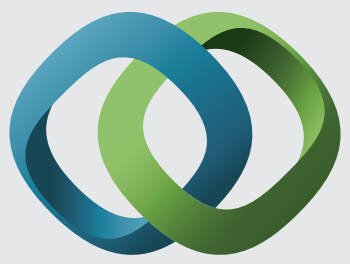

\section{Hindawi}

Submit your manuscripts at

http://www.hindawi.com
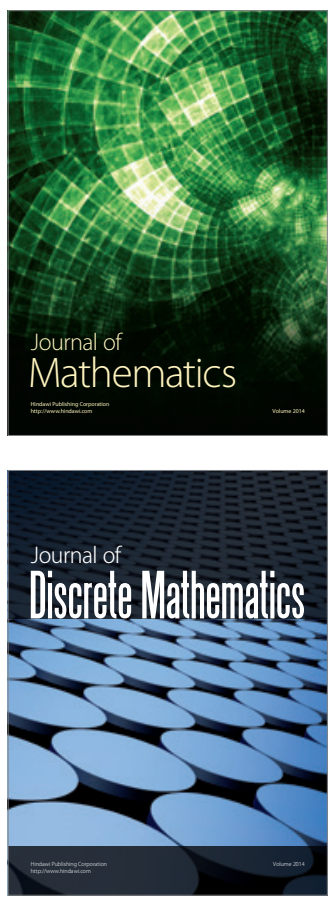

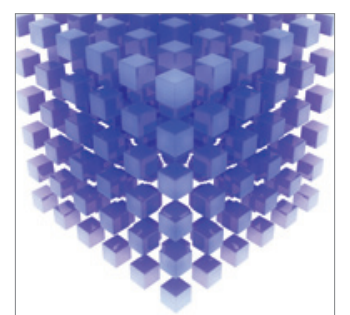

Mathematical Problems in Engineering
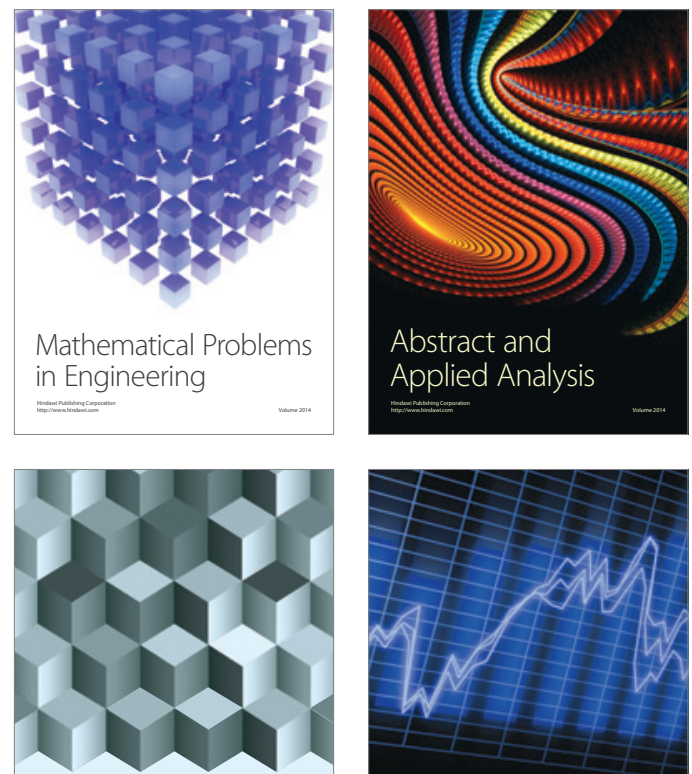

Journal of

Function Spaces

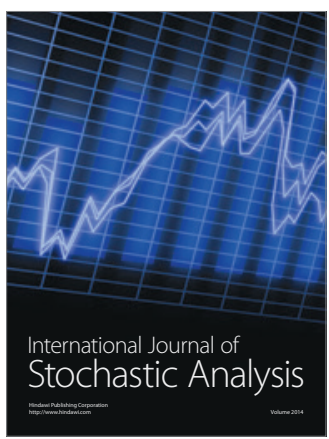

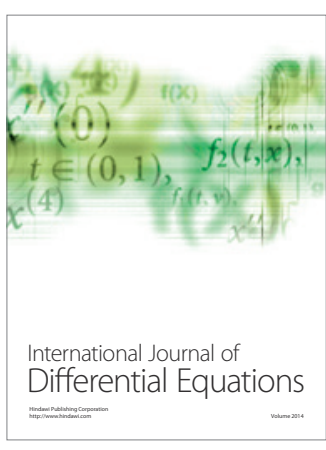
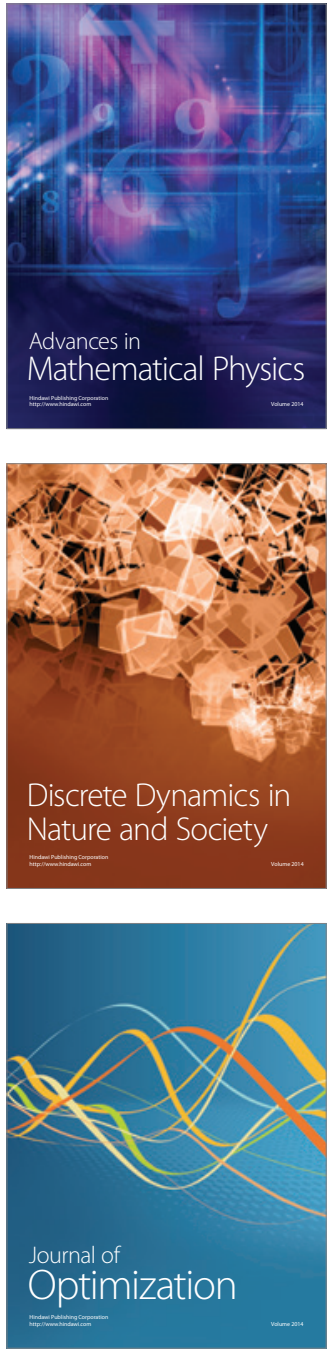